\title{
DIAGNOSTIC AND INTERVENTIONAL RADIOLOGY: A STRATEGY TO INTRODUCE REFERENCE DOSE LEVEL TAKING INTO ACCOUNT THE NATIONAL PRACTICE
}

\author{
Francis R. Verdun ${ }^{1, *}$, A. Aroua ${ }^{1}$, Ph. R. Trueb ${ }^{2}$, P. Vock ${ }^{3}$ and Jean-François Valley ${ }^{1}$ \\ ${ }^{1}$ University Institute for Applied Radiophysics, Grand-Pré 1, CH-1007 Lausanne, Switzerland \\ ${ }^{2}$ Federal Office of Public Health (FOPH), Bern, Switzerland \\ ${ }^{3}$ Universitätsspital Bern, Institut für Diagnostische Radiologie, Bern, Switzerland
}

\begin{abstract}
The purpose of this study is to present a strategy to define the reference dose levels for fluoroscopic, dose-intensive examinations. This work is a part of the project of the Federal Office of Public Health of Switzerland to translate the guidelines of the International Commission on Radiological Protection and the European Union into action. The study will also be used to set reference dose levels on the basis of a national survey. All the fluoroscopic units, involved in the survey, were equipped with a KAP (kerma-area product) meter. All KAP meters were first calibrated to ensure the comparability of the dose measurements. The doses and the dose rates together with subjective image quality measurements were acquired in all the centres. Eight types of examination were chosen by a panel of radiologists, and each of the five centres involved agreed to monitor 20 patients per examination type. A wide variation in the dose and the image quality in fixed geometry was observed. For example, the skin dose rate for abdominal examinations varied in the range of $\mathbf{1 2 - 4 2} \mathbf{m G y ~ m i n}^{-1}$ for comparable image quality. Average KAP values of 67, 178, 106, 102, 473, 205, 307 and $316 \mathrm{~Gy} \mathrm{~cm}^{2}$ were recorded for barium meal, abdominal angiography, cerebral angiography, barium enema, hepatic embolisation, biliary drainage, cerebral embolisation and femoral stenting, respectively. The values obtained in this limited study are generally higher than the ones available in the literature and strategies to optimise these studies have to be discussed. A strict control concerning the denomination of the examination type involved in such a study is mandatory to obtain reliable data. This can only be done through a close collaboration between physicians, radiographers and medical physicists.
\end{abstract}

\section{INTRODUCTION}

$\mathrm{X}$ ray examinations involving fluoroscopy, particularly those of the digestive system, angiography and interventional examinations contribute significantly to the total collective dose due to medical exposure even if their frequency is relatively low. A recent survey on the exposure of the Swiss population to $\mathrm{X}$-ray imaging indicated that this contribution amounts up to $29 \%^{(1)}$. Moreover, these types of examinations, which deliver effective doses to the patient of the order of a few $\mathrm{mSv}$ to a few tens of $\mathrm{mSv}$, can lead to extremely high entrance surface doses, up to a few Gy, leading to a significant risk of deterministic effects.

Several international bodies address seriously the issue of radiation protection of the patient and the radiologist for dose-intensive examinations. The International Commission on Radiological Protection (ICRP) dedicated one of its publications to the means and methods set up to prevent the lesions that may be induced by interventional radiology ${ }^{(2)}$. In early 2000, the Swiss Federal Office of Public Health (FOPH) formed a working group on the optimisation of radiation protection in the case of doseintensive X-ray examinations-'Optimierung des Strahlenschutzes bei dosisintensiven Untersuchungen in der Radiologie (OSUR)'. Several medical

*Corresponding author: Francis.Verdun@chuv.ch associations concerned by the issue were invited to take part in this working group: general practitioners, radiologists, cardiologists, radiographers and medical physicists. One of the main issues addressed by the working group relates to diagnostic reference levels (DRLs). In this context a study to investigate the situation of fluoroscopy in Switzerland has been launched. The aim of this study was to study the technique used to perform eight common types of examinations involving fluoroscopy in five university hospitals performing the bulk of the dose-intensive examinations, and to collect dosimetric data in order to establish DRL values for the eight types of examinations investigated. The first results of this study are reported in this paper.

\section{MATERIALS AND METHODS}

The study involved the following five Swiss hospitals (quoted as CE1-CE5 to assure anonymity): University Hospital of Lausanne, University Hospital of Geneva, Inselspital of Bern, Kantonsspital of Basel and University Hospital of Zürich, and covered the eight types of examinations summarised in Table 1: four diagnostic and four interventional procedures. The choice of these examinations has been made by the OSUR working group after long internal discussions and a wide consultation with the practitioners of the participating centres. Each centre was asked to 
Table 1. The eight types of examinations covered in the study.

\begin{tabular}{ll}
\hline Type of examination & Code \\
\hline Diagnostic & \\
Barium meal & $\mathrm{BaM}$ \\
Lower limb angiography & $\mathrm{LiA}$ \\
Cerebral angiography & $\mathrm{CeA}$ \\
Barium enema & $\mathrm{BaE}$ \\
Interventional & $\mathrm{HeE}$ \\
Hepatic embolisation & $\mathrm{BiD}$ \\
Biliary drainage and stent insertion & $\mathrm{CeE}$ \\
Cerebral embolisation & $\mathrm{IlDil}$ \\
Iliac dilatation and stent insertion & \\
\hline
\end{tabular}

register 20 examinations for each of the eight types. The centres were requested to fill in a questionnaire and provide information on the examination (fluoroscopy duration, number of images, dose-area product and the difficulty of the case), on the patient undergoing this examination (age, gender, height and weight) and on the practitioner performing the examination (medical specialty, number of years of experience and the number of examinations already performed). Moreover, the 18 fluoroscopy units used in the 5 healthcare providing centres were characterised from dose and image quality points of view. A reference KAP (kerma-area product) meter (traceable to the Swiss Federal Office of Metrology, METAS) was placed onto the KAP meter of the unit and the two readings were compared. The results were expressed in terms of KAP measured with the reference KAP meter. Entrance dose rates and entrance doses per image were then measured in the fluoroscopy and image acquisition modes, clinically in each centre, using a fixed geometry (i.e. a focus to ionisation chamber of $70 \mathrm{~cm}$, a focus to image amplifier of $1 \mathrm{~m}$, a field size at the image amplifier level of $22-25 \mathrm{~cm}$ with a $20 \mathrm{~cm}$ PMMA absorber in the field in contact with the ionising chamber) with the help of a Radcal 3036 dosemeter connected to a $11 \mathrm{~cm}^{3}$ ionising chamber (Radcal Morovia, USA). Image quality was subjectively assessed by placing a TOR CDR contrast detail test object (Leeds, UK) onto the slab of PMMA when performing dose measurements and by counting the number of low contrast targets that could be detected. If the low contrast target could be clearly distinguished a score of 1 was attributed to this target. If the target could be partially seen a score of only 0.5 was attributed. The total score, for a particular unit, was obtained by summing up the scores of the targets seen on the images. The scoring procedure, that assesses the low contrast detection performance of the unit, was performed by the same medical physicist for all the centres.

\section{RESULTS AND DISCUSSION}

One KAP meter was found to give dose indication a factor of 2 higher than the reference value. All other devices were within the $\pm 30 \%$ tolerance required by the Swiss FOPH. The results of this assessment were used to express all KAPs recorded during the survey in terms of KAPs according to the reference KAPmeter system used. A wide variation in dose and image quality (limit of low contrast detectability) in the fixed geometry chosen for the assessment of the units was observed. As an example, the skin dose rates for abdominal examinations varied in the range of $12-42 \mathrm{mGy} \mathrm{min}^{-1}$ and the image quality scores varied in the range of 4-13. It should be pointed out that for most of the systems a link between a high dose rate and a high image quality score could be established. It appeared that the most recent units using a copper filtration allowed the entrance dose to reduce significantly during fluoroscopy. Nevertheless, it was observed that this added filtration was removed during the image acquisition (e.g. DSA mode). Thus, these modern systems led to doses per image significantly higher than the ones delivered when using more conventional units. This behaviour was not expected by manufacturers who have been contacted to solve this problem.

The response rate was considered quite good since 571 examinations were registered though after a few reminders. This represents $71.4 \%$ of the total of 800 that was expected in an ideal case and corresponds to an average of 114 examinations per centre and 71 examinations per type. Some centres did not accept to give the number of examinations performed by the radiologist, information required in the form to collect the data. Thus, it was decided that radiologists with $<1$ y experience would be considered as 'trainees', radiologists with $>1$ y but $<5$ y experience would be considered as 'juniors' and radiologists with $>5$ y experience would be considered as 'seniors'. Considering the complexity of the examination, the choice between 'simple', 'standard' and 'complex' examination was left to the radiologist who performed the procedure.

Figure 1 shows the intercentre variability of the mean duration of fluoroscopy for the five types of examinations. It can be noticed that for some types there is a high variability, whereas for others the variability is relatively low. During the diagnostic examinations, the largest variability was observed for cerebral angiography in spite of the fact that these examinations were systematically performed by 'senior' radiologists. As expected, the largest variability was observed when dealing with interventional examinations, especially, for the cerebral therapeutic examination chosen (i.e. cerebral embolisation on many occasions). The assessment of dose rate and image quality showed that 
F. R. VERDUN ET AL.

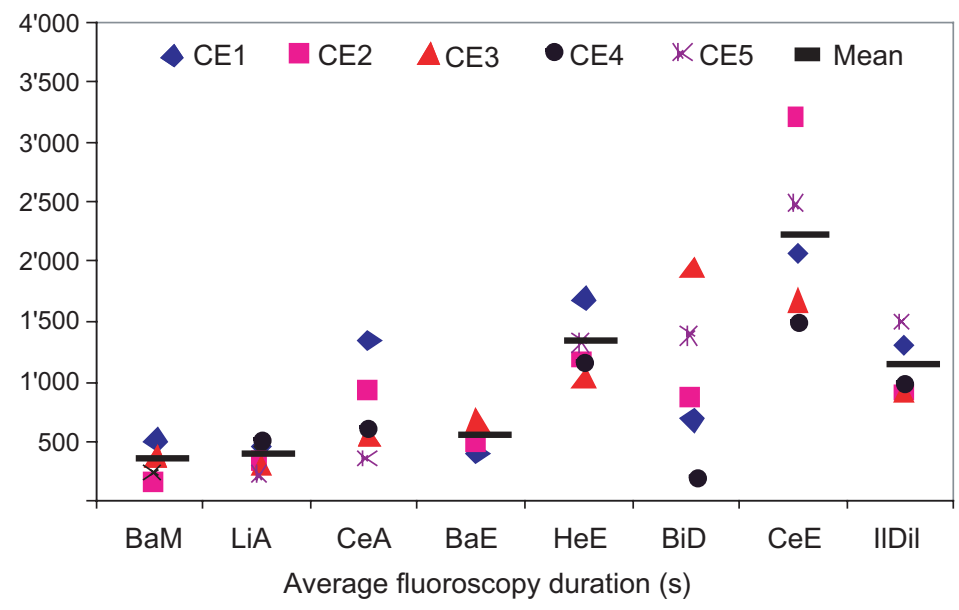

Figure 1. Intercentre variability of the fluoroscopy duration.

the systems are used within a wide range of operating points. This was mainly due by the fact that most of the radiologists or radiographers did not know that fluoroscopy systems allow the choice of the level of image quality (i.e. dose rate) one wants to use. As expected, it has been also shown that the units used for Barium studies produced the lowest image quality scores, in spite of the fact that the dose rates delivered with these systems were often superior to that measured on units used for angiography procedures. This investigation showed a strong correlation between the fluoroscopy duration and the degree of difficulty of the examination. It also showed that the number of images and fluoroscopy times were systematically higher for trainees than for junior or senior radiologists. This result clearly demonstrates that trainees need more supervision by junior or senior radiologists. DRL values were established as a function of the experience of the radiologists (three categories) and as a function of the difficulty of the examination in order to verify if these parameters introduce a significant shift in DRL. In spite of the limited statistics, it appears that in general the DRLs obtained for trainees were $30-40 \%$ higher than the ones obtained for junior and senior radiologists. It appears that the DRLs obtained for standard procedures were 2-fold greater than the ones obtained for simple procedures and 4-fold more than the ones obtained for complex procedures. In spite of these differences it was proposed to keep only one DRL per procedure, keeping in mind the range of variation that can be potentially associated with these procedures.

The KAP results obtained for all the eight types of examinations, as established from the corresponding distributions, are summarised in Table 2. Minimal, maximal, mean and 75 th percentile values of the KAP are given. The 75th percentile value corresponding to the DRL is compared to DRL values reported in the literature. The differences between DRL values can be high, particularly for complex examinations such as those performed in interventional radiology. These differences are due to several factors such as a difference in the definition of the type of examination and in the technique among the centres. The 75 th percentile to mean ratio varies from 1.05 to 1.36 with an average value of 1.24. This shows that for these examinations, if the DRLs were to be established from mean dose values, then a factor of 1.24 rather than 1.5 should be applied.

The differences between the 75th percentile values established in this work and the DRLs reported in the literature can reach a factor of 5. This should be analysed by the radiologists to see if these discrepancies are justified.

\section{CONCLUSIONS}

This study allowed the collection of data concerning 571 examinations corresponding to the 8 types. In general, all the participating centres give comparable average doses, except one for which the doses are lower by a factor of 2 than the average of the other centres. The 75th percentile values established in this work were found to be higher than the DRLs reported in the literature, sometimes by a factor of 5 . This might be due to the fact that the survey covered university hospitals exclusively, where the proportion of complex procedures might be relatively high and also there is a higher number of junior 
REFERENCE DOSE LEVELS IN FLUOROSCOPY

Table 2. KAP values for the eight types of examinations.

\begin{tabular}{lcccccrrr}
\hline & \multicolumn{7}{c}{ Examination type } \\
\cline { 2 - 8 } & BaM & LiA & CeA & BaE & HeE & BiD & CeE & IlDil \\
\hline Number of cases & 89 & 94 & 91 & 41 & 70 & 56 & 58 & 72 \\
Minimum $\left(\mathrm{Gy} \mathrm{cm}^{2}\right)$ & 3 & 8 & 3 & 20 & 54 & 5 & 24 & 36 \\
Maximum $\left(\mathrm{Gy} \mathrm{cm}^{2}\right)$ & 441 & 747 & 996 & 564 & 1703 & 1375 & 1345 & 1122 \\
Mean $\left.(\mathrm{Gy} \mathrm{cm})^{2}\right)$ & 67 & 178 & 107 & 114 & 463 & 244 & 335 & 344 \\
75th percentile $\left(\mathrm{Gy} \mathrm{cm}^{2}\right)$ & 87 & 226 & 124 & 142 & 629 & 312 & 352 & 431 \\
75th percentile $/ \mathrm{Mean}^{2}$ & 1.30 & 1.27 & 1.16 & 1.25 & 1.36 & 1.28 & 1.05 & 1.25 \\
Literature $\left(\mathrm{Gy} \mathrm{cm}^{2}\right)$ & $25^{(3)}$ & $36^{(4)}$ & $100^{(4)}$ & $60^{(3)}$ & $120^{(4)}$ & $100^{(5)}$ & - & - \\
\hline
\end{tabular}

radiologists undergoing their training. This issue should prompt a discussion between the participating radiologists.

\section{ACKNOWLEDGEMENT}

The authors are grateful to all the radiologists, radiographers and others who collaborated or provided assistance in the five participating hospitals during this survey.

\section{REFERENCES}

1. Aroua, A., Burnand, B., Decka, I., Vader J. P. and Valley, J. F. Nation-wide survey on radiation doses in diagnostic and interventional radiology in Switzerland in 1998. Health Phys. 83(1), 46-55 (2002).

2. International Commission on Radiological Protection. Avoidance of radiation injuries from medical interventional procedures. ICRP Publication 85 (Oxford: Pergamon Press) (2000).

3. Commission of the European Communities. On health protection of individuals against the dangers of ionising radiation in relation to medical exposure. Euratom Council Directive 97/43. Off. J. Eur. Commun. L180, 22-27 (1997).

4. Cruces, R. R., Garcia-Granados, J., Romero, F. J. D. and Armas, J. H. Estimation of effective dose in some digital angiographic and interventional procedures. $\mathrm{Br} . \mathrm{J}$. Radiol. 71, 42-47 (1998).

5. Padovani, R. and Maffessanti, M. Impact of EC Directive 97/43 Euratom in interventional radiology. Radiat. Prot. Dosim. 90(1-2), 39-45 (2000). 